
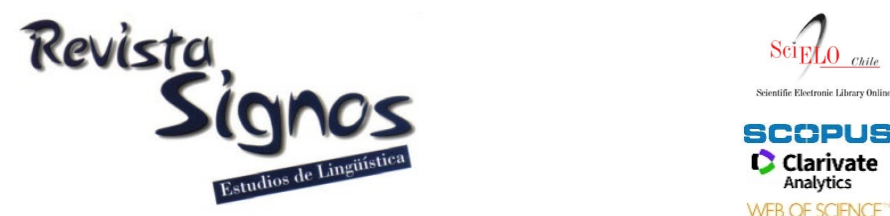

WEB OF SCFENCE

\title{
El empleo del lenguaje figurado en el discurso de personas con demencia
}

\author{
The use of figurative language in the discourse of people with \\ dementia with Alzheimer
}

\author{
Ana Varela Suárez \\ UNIVERSIDADE DE VIGO \\ ESPAÑA \\ anavarela@uvigo.es
}

Recibido: 08-I-2019 / Aceptado: 30-VIII-2019

DOI: $10.4067 /$ S0718-09342020000100272

\section{Resumen}

La mayor parte de los trabajos sobre el lenguaje figurado en el discurso de personas con demencia se ha centrado en evaluar su capacidad de comprensión (Brundage \& Bookshire, 1995; Chapman, Ulatowska, Franklin, Shobe, Thompson \& McIntire, 1997; Papagno, 2001; Amanzio, Geminiani, Leotta \& Cappa, 2008), pero son muy pocos los que han analizado cómo las personas que padecen este síndrome emplean este tipo de construcciones. Así, este trabajo presenta los siguientes objetivos: (i) identificar los diferentes tipos de lenguaje figurado que las personas con demencia emplean en su discurso; (ii) verificar si el uso del lenguaje figurado se reduce conforme avanza el deterioro cognitivo; (iii) detectar cómo el uso del lenguaje figurado puede funcionar como estrategia compensatoria para esconder los déficits discursivos causados por la demencia. Para realizar este estudio se recopiló un corpus con videos de 23 informantes con distintos tipos de demencia en diferentes estadios de la enfermedad y se han analizado los distintos tipos de lenguaje figurado que emplean, así como la frecuencia de uso de cada uno de ellos conforme avanza el deterioro cognitivo. Los resultados muestran que, aunque se produce una reducción en la frecuencia de uso, las personas con demencia emplean el lenguaje figurado hasta el final de la enfermedad. Además, recurren tanto a los extensores discursivos como al lenguaje retórico como estrategia de compensación, especialmente en el estadio moderado.

Palabras Clave: Alzheimer, lenguaje figurado, metáforas, lenguaje retórico, estrategias de compensación. 


\begin{abstract}
Most of the work conducted on figurative language in the discourse of people with dementia has been focused on their comprehension ability (Brundage \& Bookshire, 1995; Chapman, Ulatowska, Franklin, Shobe, Thompson \& McIntire, 1997; Papagno, 2001; Amanzio, Geminiani, Leotta \& Cappa, 2008). However, there are not many studies analyzing how people suffering from this syndrome introduce this type of constructions. Bearing this in mind, this work presents the following aims: (i) to identify the different types of figurative language people with dementia use; (ii) to verify whether the frequency of use of figurative language decreases as the cognitive decline progresses; (iii) to detect how the use of figurative language can work as a compensatory strategy to hide the deficits caused by dementia. In order to do so, a corpus with videos from 23 people suffering from different forms of dementia and in different stages of the disease was compiled. The types of figurative language they introduce as well as their frequency of use have been analyzed. The results show that, even if there is a decline in their frequency of use, people with dementia still utilize figurative language until the final stage of the disease. Moreover, they resort to both discursive extenders and rhetoric language as a compensatory strategy, particularly during the moderate stage.
\end{abstract}

Key Words: Alzheimer, figurative language, metaphors, rhetoric language, compensatory strategies.

\title{
INTRODUCCIÓN
}

Dentro del campo de la pragmática clínica, una de las poblaciones más estudiadas son las personas con demencia. Esto se debe, por un lado, a su alta tasa de prevalencia (5-8\% de las personas mayores de 60 años a nivel mundial (Organización Mundial de la Salud, 2017)) y, por otro, a que este grupo presenta alteraciones pragmáticas en el discurso desde prácticamente el comienzo de la enfermedad (véase, por ejemplo, Lubinski, 1995; Perkins, Whitworth \& Lesser, 1998; Perkins, 2008; Davis \& Guendouzi, 2013; Pérez Mantero, 2017; Varela Suárez, 2018). Sin embargo, uno de los grandes olvidados dentro de las cuestiones objeto de estudio de este ámbito ha sido el lenguaje figurado.

Hasta la fecha, la mayor parte de los estudios que se han publicado sobre este tema se han centrado en la comprensión de lenguaje figurado en personas que padecen la Enfermedad de Alzheimer (EA). No obstante, en lo que respecta a la producción, el número de investigaciones que conocemos es bastante más limitado. Es por ello que en este trabajo hemos seleccionado como objeto de estudio la producción del lenguaje figurado en el discurso de las personas que padecen demencia. Para ello, partimos de los siguientes objetivos:

(i) Identificar los diferentes tipos de lenguaje figurado que las personas con demencia emplean en su discurso. 
(ii) Verificar si el uso del lenguaje figurado se reduce conforme avanza el deterioro cognitivo.

(iii)Detectar cómo el uso del lenguaje figurado puede funcionar como estrategia compensatoria para esconder los déficits discursivos causados por la demencia.

Puesto que la incidencia de las alteraciones del lenguaje en la demencia se ha cifrado entre un $88 \%$ y un $95 \%$, y la mayor parte de los trabajos sobre pragmática han determinado un deterioro en la producción oral (véase, por ejemplo, Hamilton 1994a; Causino Lamar, Obler, Knoefel \& Albert, 1994; Chapman, Highley \& Thompson, 1998; March, Wales \& Pattinson, 2003; Rousseaux, Sève, Vallet, Pasquier \& Mackowiak-Cordoliani, 2010), la hipótesis de partida de este trabajo es que tanto la construcción correcta como la frecuencia de uso del lenguaje figurado se deteriorará conforme avanza la demencia.

Así, en las líneas que siguen presentaremos, en primer lugar, el estado de la cuestión, en el que repasaremos las aportaciones más relevantes publicadas hasta la fecha sobre el lenguaje figurado y la demencia, así como sobre la competencia estratégica y la demencia; a continuación, detallaremos la metodología que hemos aplicado para realizar este estudio; a seguir, mostraremos los resultados obtenidos y, por último, cerraremos con los apartados de discusión y conclusiones, en los que analizaremos los resultados del trabajo.

\section{Estado de la cuestión}

\subsection{Lenguaje figurado}

Tal y como apuntamos en el apartado anterior, la mayor parte de los trabajos sobre lenguaje figurado y demencia se han centrado en la capacidad de comprensión. Los resultados de estas aportaciones apuntan a que la comprensión del lenguaje figurado que a los enfermos de Alzheimer les resulta familiar y escuchan con frecuencia no suele ser problemática (Papagno, 2001). Sin embargo, aquellas expresiones menos familiares y/o infrecuentes suelen interpretarlas por su sentido literal (Brundage \& Bookshire, 1995; Chapman et al., 1997; Amanzio et al., 2008). Además, se ha detectado que la capacidad para comprender las metáforas y el sarcasmo se deteriora conforme avanza el deterioro cognitivo (Maki, Yamaguchi, Koeda \& Yamaguchi, 2013).

En lo que concierne a la producción oral, en cambio, el número de aportaciones ha sido considerablemente más escaso. El trabajo más relevante que hemos encontrado hasta la fecha es el de Maclagan, Davis y Lunsford (2008), quienes apuntaron que la capacidad para construir secuencias basadas en el uso del lenguaje figurado se conserva conforme avanza el deterioro cognitivo y que, de hecho, muchas veces es empleado como estrategia compensatoria para mantener la competencia comunicativa. Asimismo, también encontramos algunos trabajos que han considerado el uso de los 
extensores o expresiones formulaicas en casos de EA y han concluido que el empleo de tales estrategias es más recurrente en las personas con demencia que en los controles (Bridges \& Van Lancker Sidtis, 2013; Zimmerer, Wibrow \& Varley, 2016). Con respecto a esto, en su trabajo Wray (2011) presentó un modelo con el que comprobó que introducir estas expresiones formulaicas puede ser un impedimento en la comunicación entre las personas con Alzheimer y quienes las cuidan.

\subsection{Competencia estratégica}

A diferencia del lenguaje figurado, la capacidad que muestran las personas con demencia para utilizar la competencia estratégica, es decir, la habilidad de recurrir a estrategias discursivas para disimular los problemas del lenguaje, ha sido el foco de estudio de numerosos trabajos. Uno de los primeros y más relevantes es el de Hamilton (1994b), que documentó que Elsie, la informante de aquel estudio, en las fases iniciales en las que todavía era consciente de su enfermedad, pedía frecuentemente aclaraciones sobre la conversación como estrategia para disimular la confusión que sentía. No obstante, conforme la enfermedad fue avanzando, Elsie pedía cada vez menos aclaraciones, hacía menos referencia a sus problemas de memoria y menos autoevaluaciones, lo que para la autora es un reflejo de la pérdida de la capacidad de la enferma para evaluar su propio deterioro del lenguaje.

Orange, Lubinski y Higginbotham (1996) analizaron la capacidad para reparar el discurso de personas con demencia leve y moderada, y descubrieron que, a medida que avanza la enfermedad, se crean más problemas discursivos y, por lo tanto, la necesidad de repararlos aumenta. Además, también se dieron cuenta de que las personas con demencia moderada, así como sus interlocutores, emplean más términos inespecíficos para evitar los malentendidos. Por su parte, Ripich, Ziol, Fritsch y Durand (2000) afirman que conforme avanza la enfermedad aumenta la necesidad de emplear estrategias pragmáticas compensatorias, como preguntas y enunciados dubitativos, lo que para los autores demuestra que la intención de comunicar se mantiene a lo largo de toda la enfermedad.

En su investigación, Guendouzi y Müller (2002) comprobaron que una estrategia de reparación útil era hablar sobre temas conocidos y con los que se sienten cómodos la persona con demencia. También insistieron en la necesidad de revisar el término 'conversación satisfactoria' (desde la perspectiva griceana), pues para una persona con EA esta satisfacción se rige por unos parámetros diferentes. Además, Vasse, VernooijDassen, Spijker, Rikkert y Koopmans (2010) revisaron 19 estudios de intervención y se dieron cuenta de que mediante la realización de actividades diarias y sesiones con una única tarea sus informantes desarrollaban estrategias que les permitían mejorar la comunicación con el personal que los trataba y cuidaba. 
Por último, Varela Suárez (2017) revisó cuáles eran las estrategias que empleaban 12 personas con demencia para reaccionar en el par adyacente pregunta-respuesta. La autora concluyó que el estadio en el que se hacía más uso de estas estrategias era el moderado, pues en el inicial todavía no resultaban tan necesarias y en el avanzado la habilidad para recurrir a ellas comenzaba a deteriorarse. Las estrategias a las que recurrieron sus informantes con más asiduidad fueron responder con preguntas de confirmación, dar respuestas generales a preguntas específicas y admitir abiertamente el déficit comunicativo para justificar la incapacidad para dar una respuesta.

\section{Marco metodológico}

\subsection{Participantes}

La Asociación de Familiares de Enfermos de Alzheimer de Galicia (AFAGA) nos proporcionó una lista de personas que se presentaron voluntarias para participar en la presente investigación. A esta lista inicial se le aplicaron los siguientes criterios de inclusión:

a) Presentar un diagnóstico de alguna de las formas de demencia.

b) Aportar una hoja de consentimiento informado firmado por el cuidador a cargo.

c) Tener como lengua materna el gallego y/o el castellano.

Además, se determinaron los siguientes criterios de exclusión:

a) Padecer déficits audiovisuales que puedan sesgar los resultados de la prueba.

b) Presentar trastornos de la conducta que impidan desarrollar la prueba con normalidad.

c) Presentar un trastorno de afasia severa que dificulte la identificación de palabras y frases para su posterior análisis.

Una vez aplicados estos criterios, la lista definitiva de participantes constó de 23 personas. Todos los datos relativos a su historia personal y perfil neuropsicológico fueron proporcionados por informes que elaboró el personal de AFAGA. Así, la edad media de la muestra fue de 80 años $(\mathrm{DE}=8,0016)$. La distribución por tipo de demencia fue de: 17 personas con EA (73,9\%), 3 con demencia mixta (13\%), 1 con demencia vascular $(4,3 \%), 1$ con demencia frontotemporal $(4,3 \%)$ y 1 con deterioro cognitivo sin especificar (4,3\%). La distribución por grado de deterioro cognitivo se basó en la aplicación de la Escala de Deterioro Global de Reisberg (GDS) y fue de 4 personas con GDS-3 (17,4\%), 8 con GDS-4 (34,8\%), 6 de GDS-5 (26,1\%), 2 de GDS-6 (8,7\%) y 3 de GDS-7 (13\%).

En lo que concierne a la distribución por género, 15 eran mujeres $(65,2 \%)$ y 8 hombres $(34,8 \%)$, y en lo que respecta a la primera lengua, 15 eran hablantes de 
castellano $(65,2 \%)$ y 8 de gallego (34,8\%). A modo de resumen, en la Tabla 1 se recogen todos los datos de las personas que han participado en esta investigación.

Tabla 1. Datos de las personas participantes.

\begin{tabular}{|c|c|c|c|c|c|}
\hline Informante & Diagnóstico & GDS & Edad & Lengua & Género \\
\hline I01 & EA & 7 & 90 & Castellano & Mujer \\
\hline I02 & EA & 7 & 77 & Gallego & Mujer \\
\hline I03 & EA & 7 & -1 & Castellano & Mujer \\
\hline I04 & Dem. mixta & 6 & 90 & Gallego & Hombre \\
\hline I05 & EA & 6 & 61 & Castellano & Hombre \\
\hline I06 & EA & 5 & 90 & Gallego & Mujer. \\
\hline I07 & EA & 5 & 86 & Gallego & Mujer \\
\hline I08 & EA & 5 & 73 & Gallego & Hombre \\
\hline I09 & Dem. mixta & 5 & 86 & Gallego & Hombre \\
\hline I10 & Dem. vascular & 5 & 86 & Castellano & Mujer \\
\hline I11 & Dem. sin especificar & 5 & - & Gallego & Mujer \\
\hline I12 & EA & 4 & 89 & Castellano & Mujer \\
\hline I13 & EA & 4 & 83 & Castellano & Mujer \\
\hline I14 & EA & 4 & 85 & Gallego & Mujer \\
\hline I15 & EA & 4 & - & Castellano & Mujer \\
\hline I16 & EA & 4 & 69 & Castellano & Mujer \\
\hline I17 & EA & 4 & 76 & Castellano & Hombre \\
\hline I18 & EA & 4 & - & Castellano & Mujer \\
\hline I19 & EA & 4 & 80 & Castellano & Hombre \\
\hline I20 & EA & 3 & 74 & Castellano & Hombre \\
\hline I21 & EA & 3 & 79 & Castellano & Hombre \\
\hline I22 & Dem. frontotemporal & 3 & 79 & Castellano & Mujer \\
\hline I23 & Dem. mixta & 3 & 72 & Castellano & Mujer \\
\hline
\end{tabular}

\subsection{Recopilación del corpus}

Para poder analizar la producción de lenguaje figurado de forma espontánea, el formato elegido para los textos del corpus fue una entrevista sobre la vida, los gustos y las rutinas de las personas participantes, pues se consideró que era el tipo de narración con el que se iban a sentir más cómodas y, por lo tanto, el lenguaje figurado iba a surgir de forma más espontánea.

De este modo, se grabó a las 23 personas participantes en vídeo con una cámara SONY Handycam HD AVCHD, con el objetivo de poder tener en cuenta también el lenguaje no verbal durante el análisis. Todas las entrevistas se realizaron en las instalaciones de la AFAGA, con la excepción de aquellas personas con problemas de movilidad, a las que se grabó en su domicilio.

Todas las grabaciones se llevaron a cabo entre abril y mayo de 2016 y su duración varió entre 6 y 30 minutos, en función de la voluntad de hablar de las personas entrevistadas. El idioma elegido para hacer la grabación fue en todos los casos la 
primera lengua. Al comienzo de la grabación, todas fueron informadas de que iban a ser grabadas en vídeo mientras mantenían una conversación con la entrevistadora. A continuación, se muestran algunos ejemplos de las preguntas que se plantearon durante las grabaciones:

¿Cuál es su nombre completo?

¿Dónde nació?

¿Dónde vive actualmente?

¿Le gusta la ciudad en la que vive?

¿Qué es lo que más le gusta de su barrio?

¿Con quién vive actualmente?

¿Tiene hijos/hijas? ¿Cómo se llaman?

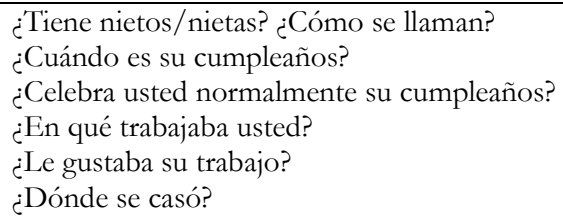

\subsection{Transcripción y anotación}

La transcripción del corpus se realizó a través del software ELAN y se empleó una adaptación del sistema de pautas propuesto por Müller (2006):

\begin{tabular}{|l|l|}
\hline I01 & $\begin{array}{l}\text { Designa a la persona informante en el corpus. En la anotación se emplea para anunciar sus } \\
\text { intervenciones. }\end{array}$ \\
\hline INV & Anuncia las intervenciones de la entrevistadora. \\
\hline NV & Anuncia las intervenciones no verbales. \\
\hline$:$ & El sonido que precede a este símbolo ha sido alargado \\
\hline- & La palabra a la que precede se ha cortado de forma brusca y se ha sustituido por otra. \\
\hline $\mathrm{Xx}$ & Palabra o sonido no reconocible. \\
\hline$\ldots$ & Enunciado sin terminar. \\
\hline$(2.5)$ & Tiempo entre enunciados cuando la pausa es superior a 20ms. \\
\hline
\end{tabular}

Todas las transcripciones fueron revisadas por una segunda persona.

En lo que respecta a la anotación, este trabajo se centró en los siguientes cinco tipos de lenguaje figurado: expresiones fijas, extensores, metonimias, metáforas y lenguaje retórico. De este modo, en primer lugar, se clasificaron todos los enunciados que incluían alguna de estas formas de lenguaje figurado según su tipo correspondiente y, a continuación, se clasificó cada uno de ellos con una de estas tres etiquetas: (i) uso correcto, (ii) error en la estructura, (iii) uso en un contexto incorrecto (error de tipo pragmático). Con el objetivo de facilitar la comprensión del sistema de etiquetado, mostramos la Tabla 2, con ejemplos de cada uno de los tipos de etiquetas que se utilizó en esta investigación. 
Tabla 2. Explicación y ejemplos de la clasificación empleada en esta investigación.

\begin{tabular}{|c|c|c|}
\hline Etiqueta & $\begin{array}{l}\text { Valor } \\
\end{array}$ & Ejemplo \\
\hline $\begin{array}{l}\text { Expresión fija } \\
\text { correcta }\end{array}$ & $\begin{array}{l}\text { El hablante introduce una expresión fija, es } \\
\text { decir, formas invariables como refranes, dichos } \\
\text { o colocaciones de forma correcta. }\end{array}$ & $\begin{array}{l}\text { Lo tengo todo muy a mano. Yo soy de } \\
\text { La Voz de toda la vida. }\end{array}$ \\
\hline $\begin{array}{l}\text { Expresión fija } \\
\text { error de estructura }\end{array}$ & $\begin{array}{l}\text { El hablante introduce una "expresión fija" pero } \\
\text { la construye de forma incorrecta. }\end{array}$ & $\begin{array}{l}\text { Fuimos allá a Santander y estamos en } \\
\text { una piña_todos. }\end{array}$ \\
\hline $\begin{array}{l}\text { Expresión fija } \\
\text { error pragmático. }\end{array}$ & $\begin{array}{l}\text { El hablante introduce una expresión fija en el } \\
\text { contexto incorrecto. }\end{array}$ & $\begin{array}{l}\text { INV: ¿Ya son mayores (sus nietos)? } \\
\text { - Ya están en la edad, sí. }\end{array}$ \\
\hline Extensor correcto & $\begin{array}{l}\text { El hablante introduce un "extensor", es decir, } \\
\text { una frase con sentido general con el objetivo } \\
\text { de rellenar el discurso. }\end{array}$ & $\begin{array}{l}\text { INF: Era ingeniero de caminos } \\
\text { - ¡Caray! } \\
\text { - Sí. Y muy bien. Y entonces empezó } \\
\text { asi con-con las piernas que le fallaron y } \\
\text { con esas cosas y... }\end{array}$ \\
\hline $\begin{array}{l}\text { Extensor error de } \\
\text { estructura }\end{array}$ & $\begin{array}{l}\text { El hablante introduce un extensor discursivo } \\
\text { con una estructura incorrecta. }\end{array}$ & No hay ejemplos en el corpus \\
\hline $\begin{array}{l}\text { Extensor error } \\
\text { pragmático. }\end{array}$ & $\begin{array}{l}\text { El hablante introduce un extensor en el } \\
\text { contexto incorrecto. }\end{array}$ & $\begin{array}{l}\text { Entón ao mellor vas ti pum-pum-pum } \\
\text { [habla sobre el presente]. A vida } \\
\text { era así. }\end{array}$ \\
\hline $\begin{array}{l}\text { Metonimia } \\
\text { correcta }\end{array}$ & $\begin{array}{l}\text { El hablante utiliza una metonimia, es decir, la } \\
\text { denominación de una parte de un concepto } \\
\text { para referirse al todo, de forma correcta }\end{array}$ & $\begin{array}{l}\text { Bueno, cualquiera, a mi me va } \\
\text { cualquier plato. }\end{array}$ \\
\hline $\begin{array}{l}\text { Metonimia error } \\
\text { de estructura }\end{array}$ & $\begin{array}{l}\text { El hablante intenta introducir una metonimia, } \\
\text { pero la estructura incorrectamente. }\end{array}$ & No hay ejemplos en el corpus \\
\hline $\begin{array}{l}\text { Metonimia error } \\
\text { pragmático. }\end{array}$ & $\begin{array}{l}\text { El hablante utiliza una metonimia en el } \\
\text { contexto incorrecto. }\end{array}$ & No hay ejemplos en el corpus \\
\hline Metáfora correcta & $\begin{array}{l}\text { El hablante introduce una metáfora, es decir, } \\
\text { emplea un concepto en lugar de otro para } \\
\text { sugerir algún tipo de analogía entre ellos. }\end{array}$ & $\begin{array}{l}\text { Nos vemos de pascuas en flores. } Y \\
\text { desde que faltó mi mama menos. }\end{array}$ \\
\hline $\begin{array}{l}\text { Metáfora error de } \\
\text { estructura }\end{array}$ & $\begin{array}{l}\text { El hablante intenta emplear una metáfora, pero } \\
\text { la construye de forma incorrecta. }\end{array}$ & $\begin{array}{l}\text { Pues mira, mi hermana aprendió a } \\
\text { coser. Yo... Pues... Eran aquellos } \\
\text { tiempos asi más... Terminales. }\end{array}$ \\
\hline $\begin{array}{l}\text { Metáfora error } \\
\text { pragmático. }\end{array}$ & $\begin{array}{l}\text { El hablante emplea una metáfora, pero la } \\
\text { introduce en el contexto incorrecto. }\end{array}$ & No hay ejemplos en el corpus \\
\hline $\begin{array}{l}\text { Lenguaje retórico } \\
\text { correcto }\end{array}$ & $\begin{array}{l}\text { El hablante hace uso del sarcasmo, ironía, } \\
\text { retranca o introduce una pregunta retórica. }\end{array}$ & $\begin{array}{l}\text { INV: E ten fillos? [...] } \\
\text { - Eu non, a muller tuvo. }\end{array}$ \\
\hline $\begin{array}{l}\text { Lenguaje retórico } \\
\text { error de estructura }\end{array}$ & $\begin{array}{l}\text { El hablante intenta hacer uso del sarcasmo, } \\
\text { ironía, retranca o introduce una pregunta } \\
\text { retórica pero la estructura que emplea no es } \\
\text { comprensible. }\end{array}$ & No hay ejemplos en el corpus \\
\hline $\begin{array}{l}\text { Lenguaje retórico } \\
\text { error pragmático. }\end{array}$ & $\begin{array}{l}\text { El hablante intenta hacer uso del sarcasmo, } \\
\text { ironía, retranca o introduce una pregunta } \\
\text { retórica pero no lo contextualiza } \\
\text { adecuadamente. }\end{array}$ & No hay ejemplos en el corpus \\
\hline
\end{tabular}

\subsection{Tratamiento de los datos}

Para poder cumplir el objetivo (ii) de esta investigación (verificar si el uso recurrente del lenguaje figurado se reduce conforme avanza el deterioro cognitivo), se emplearon tanto el coeficiente de correlación de Pearson, que mide la relación lineal entre dos variables (Murgiondo \& Tejedor, 2005) como el coeficiente de determinación $\left(R^{2}\right)$, que indica el porcentaje de variabilidad de los datos que resulta al 
poner en relación dos variables (Pita Fernández \& Pértega Díaz, 1997). Ambos análisis se realizaron con las variables 'porcentaje de usos correctos' y 'estadio de la enfermedad'. Todos los análisis estadísticos se realizaron mediante el programa IBM SPSS Statistics 23.

\section{Resultados}

\subsection{Frecuencia de uso}

En total en este trabajo se han identificado 665 enunciados en los que se ha empleado el lenguaje figurado. La forma más empleada por las personas que han participado en este estudio han sido las expresiones fijas (230), seguidas de los extensores (150) y las metáforas (150). Las metonimias (72) y el lenguaje retórico (42) han sido menos frecuentes. Si atendemos al uso del lenguaje figurado contando las expresiones únicamente la primera vez que el informante las emplea, es decir, eliminando las repeticiones, nos encontramos con que las formas más utilizadas siguen siendo las expresiones fijas (185), seguidas de las metáforas (126), de los extensores (80), de las metonimias (57) y, por último, las figuras retóricas (38). De este modo, la forma en la que se han dado más repeticiones han sido los extensores $(46,6 \%$ del total), seguidos de las metonimias (20,8\%) y de las expresiones fijas (19,6\%). Las formas menos repetidas han sido las metáforas (16\%) y el lenguaje retórico $(9,5 \%)$. El Gráfico 1 muestra la frecuencia de uso de las distintas formas con y sin repeticiones.

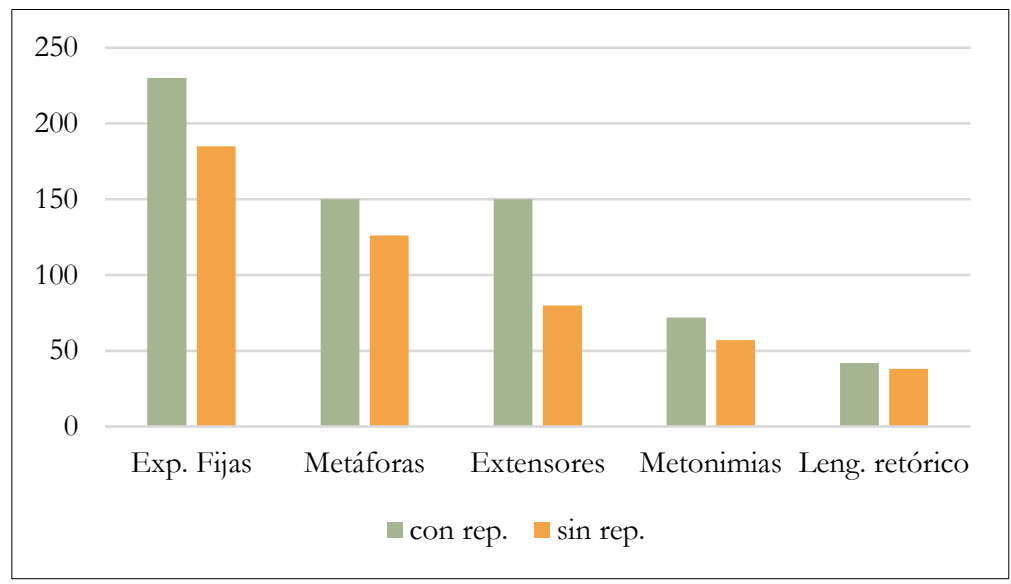

Gráfico 1. Frecuencia de uso de las distintas formas de lenguaje figurado con y sin repeticiones.

\subsection{Usos incorrectos}

De los 665 enunciados etiquetados como lenguaje figurado, tan solo se detectaron 20 formas erróneas, es decir, el 3\% del total. La forma de error más común fueron las expresiones fijas utilizadas en un contexto inapropiado (45\%), seguidas de metáforas construidas incorrectamente (35\%), expresiones fijas construidas incorrectamente 
$(15 \%)$ y extensores empleados en el contexto inapropiado (5\%). El Gráfico 2 ilustra los tipos de errores de lenguaje figurado encontrados dentro del corpus.

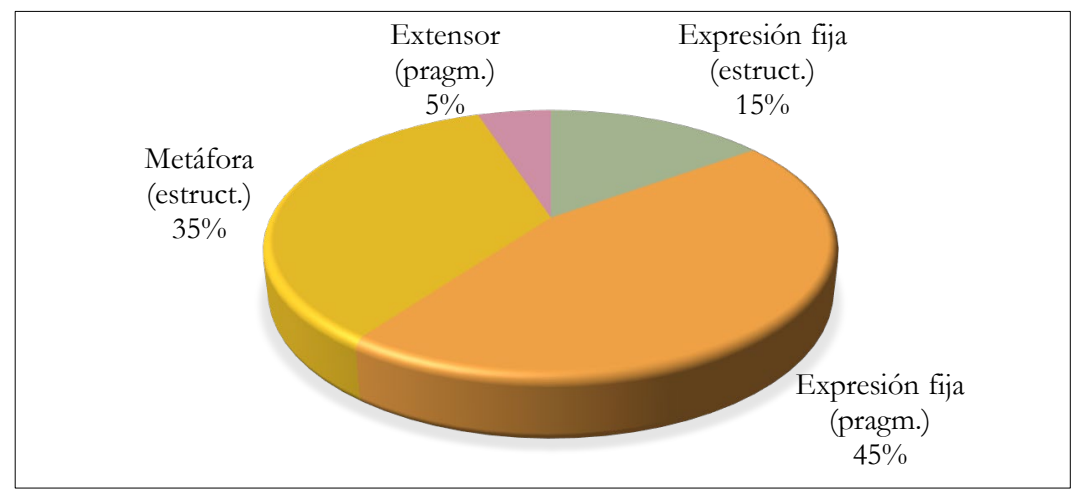

Gráfico 2. Porcentajes de tipos de uso incorrecto del lenguaje figurado.

\subsection{Lenguaje figurado y deterioro cognitivo}

Con el objetivo de poder determinar si el uso del lenguaje figurado empeora conforme avanza el deterioro cognitivo de las personas con demencia, se creó un ratio de expresiones por minuto debido a la diferencia de longitud de las grabaciones de los distintos informantes. Los resultados indican que existe una correlación negativa entre el estadio de la enfermedad (GDS-3 y GDS-4= leve, GDS-5= moderado; GDS- 6 y GDS-7=severo $)(M=1,70 ; \mathrm{DE}=0,82)$ y el ratio de expresiones con lenguaje figurado correctas por minuto $(\mathrm{M}=1,64 ; \mathrm{DE}=1,04), r=-, 605, p \leq .002, \mathrm{n}=23$.

Los informantes con GDS-3 emitieron 1,5 expresiones con lenguaje figurado por minuto, los de GDS-4 1,6, los de GDS-5 1,2, los de GDS-6 0,4 y los de GDS-7 0,3. Si englobamos esta información en los tres estadios de la enfermedad, obtenemos que en el estadio leve la media fue de 1,6 expresiones con lenguaje figurado por minuto; en el estadio moderado esta cifra descendió un $25 \%$ (1,2 exp./min.) y en el estadio severo un 56,25\% (0,3 exp./min.). El Gráfico 3 ilustra las expresiones con lenguaje figurado por minuto emitidas por los informantes divididos por grado en la escala GDS. El Gráfico 4 muestra las expresiones con lenguaje figurado por minuto emitidas por los informantes divididos por estadio de la enfermedad. 


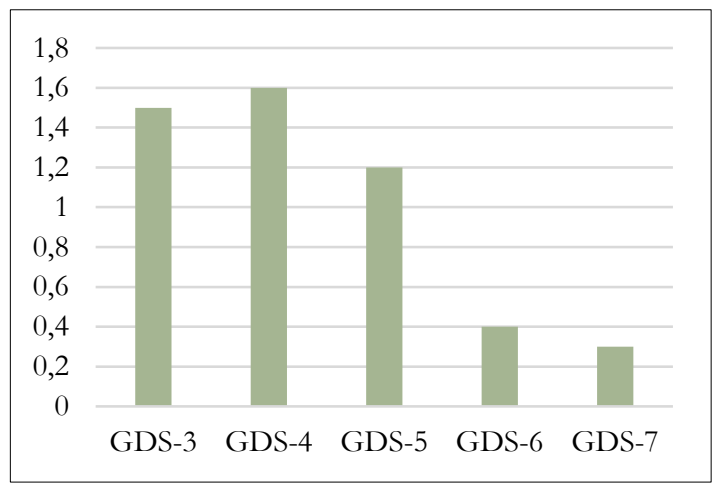

Gráfico 3. Expresiones con lenguaje figurado por minuto emitidas por los informantes clasificados por GDS.

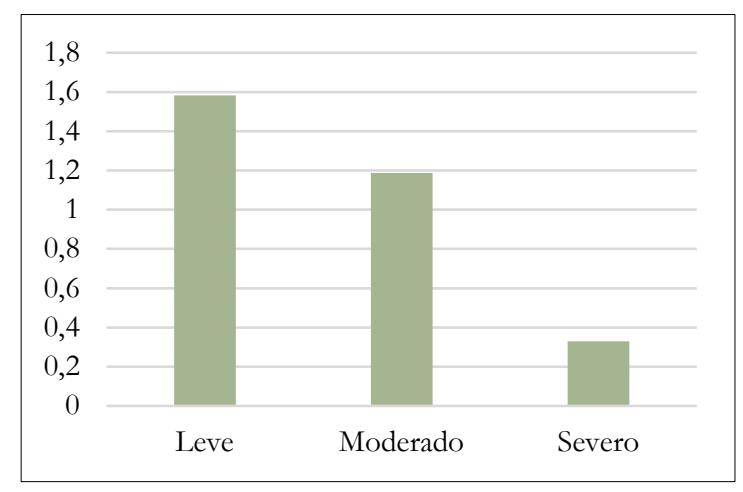

Gráfico 4. Expresiones con lenguaje figurado por minuto emitidas por los informantes de cada estadio.

La Tabla 3 muestra el ratio por minuto del uso de expresiones con lenguaje figurado por parte de los informantes. En los apartados que siguen se desglosa la información del uso de cada tipo de lenguaje figurado identificado en este trabajo.

Tabla 3. Ratio por minuto del uso de expresiones con lenguaje figurado por parte de los informantes.

\begin{tabular}{|c|c|c|c|c|c|c|}
\hline Det. cog. & Exp. fijas & Extensores & Metonimias & Metáforas & Leng. retórico & Total \\
\hline GDS-7 & 0,1 & 0 & 0,0 & 0,0 & 0,0 & 0,3 \\
\hline GDS-6 & 0,2 & 0,1 & 0 & 0,1 & 0 & 0,4 \\
\hline GDS-5 & 0,4 & 0,2 & 0,1 & 0,2 & 0,3 & 1,2 \\
\hline GDS-4 & 0,6 & 0,2 & 0,2 & 0,4 & 0,1 & 1,6 \\
\hline GDS-3 & 0,5 & 0,3 & 0,2 & 0,5 & 0,1 & 1,5 \\
\hline Severo & 0,2 & 0,1 & 0 & 0,1 & 0 & 0,3 \\
\hline Moderado & 0,4 & 0,2 & 0,1 & 0,2 & 0,3 & 1,2 \\
\hline Leve & 0,6 & 0,2 & 0,2 & 0,4 & 0,1 & 1,6 \\
\hline
\end{tabular}




\subsubsection{Expresiones fijas}

Los resultados indican que existe una correlación negativa entre el estadio de la enfermedad (GDS-3 y GDS-4= leve, GDS-5= moderado; GDS-6 y GDS-7=severo) $(\mathrm{M}=1,70 ; \mathrm{DE}=, 82)$ y el ratio de expresiones fijas correctas por minuto $(\mathrm{M}=, 47$; $\mathrm{DE}=, 34), r=-, 539, p \leq .008, \mathrm{n}=23$. Los informantes con GDS-3 emitieron de media 0,5 expresiones fijas por minuto, los de GDS-4 0,7, los de GDS-5 0,5, los de GDS-6 0,2 y los de GDS-7 0,2. Si atendemos a los datos distribuidos por estadios de la enfermedad, obtenemos que en el estadio leve la media fue de 0,6 expresiones fijas por minuto, en el estadio moderado esta cifra descendió un $44,4 \%(0,4$ exp. $/ \mathrm{min}$.) y en el estadio severo un 50\% (0,2 exp./min.). El Gráfico 5 ilustra las expresiones fijas por minuto emitidas por los informantes divididos por grado en la escala GDS. El Gráfico 6 muestra las expresiones fijas por minuto emitidas por los informantes divididos por estadio de la enfermedad.

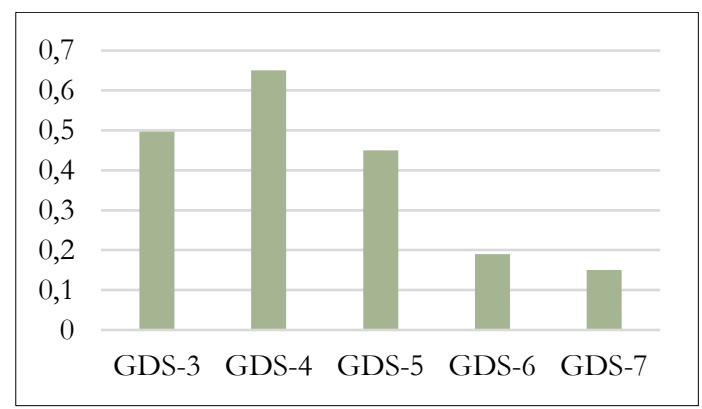

Gráfico 5. Expresiones fijas por minuto emitidas por los informantes clasificados por GDS.

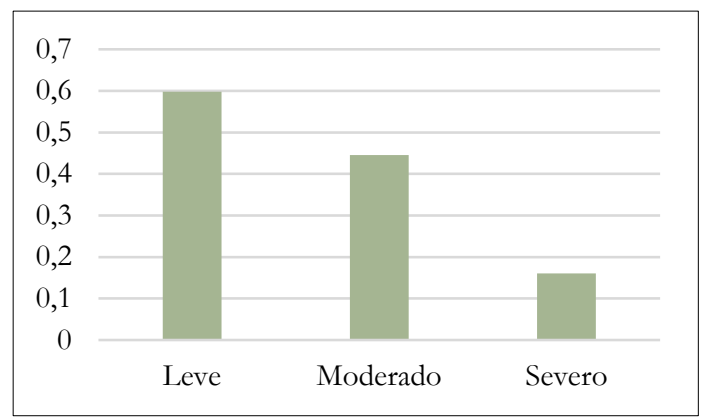

Gráfico 6. Expresiones fijas por minuto emitidas por los informantes de cada estadio.

\subsubsection{Extensores discursivos}

Los resultados apuntan a que existe una correlación negativa entre el estadio de la enfermedad (GDS-3 y GDS-4= leve, GDS-5= moderado; GDS-6 y GDS-7=severo) $(\mathrm{M}=1,70 ; \mathrm{DE}=, 82)$ y el ratio de extensores discursivos correctos por minuto $(\mathrm{M}=, 18$; $\mathrm{DE}=, 14), r=-, 521, p \leq .011, \mathrm{n}=23$. Los informantes con GDS-3 emitieron de media 
0,3 extensores por minuto, los de GDS-4 0,2, los de GDS-5 0,2, los de GDS-6 0,1 y los de GDS-7 0. Si atendemos a los datos distribuidos por estadios de la enfermedad, obtenemos que en el estadio leve la media fue de 0,25 extensores por minuto, en el estadio moderado esta cifra descendió un 20\% (0,2 ext./min.) y en el estadio severo un 40\% (0,1 ext./min.). El Gráfico 7 ilustra los extensores por minuto emitidos por los informantes divididos por grado en la escala GDS. El Gráfico 8 muestra los extensores por minuto emitidos por los informantes divididos por estadio de la enfermedad.

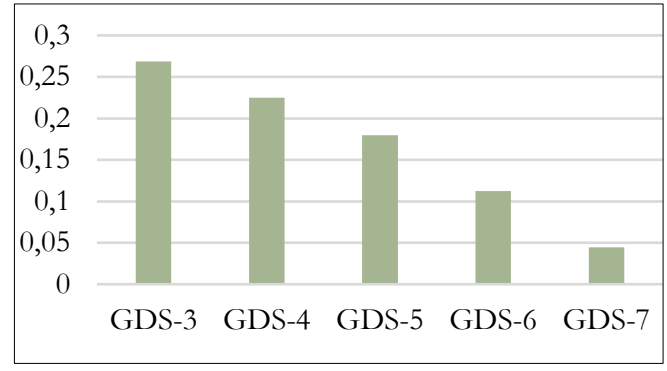

Gráfico 7. Extensores por minuto emitidos por los informantes clasificados por GDS.

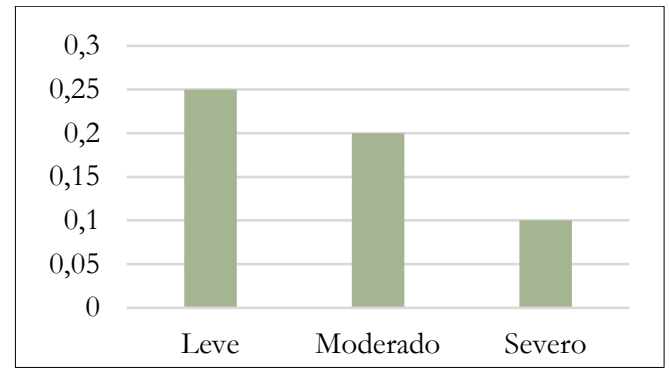

Gráfico 8. Extensores por minuto emitidas por los informantes de cada estadio.

\subsubsection{Metonimias}

Los resultados señalan que existe una correlación negativa entre el estadio de la enfermedad (GDS-3 y GDS-4= leve, GDS-5= moderado; GDS-6 y GDS-7=severo) $(\mathrm{M}=1,70 ; \mathrm{DE}=, 82)$ y el ratio de metonimias correctas por minuto $(\mathrm{M}=, 14 ; \mathrm{DE}=, 13)$, $r=-, 759, p_{<}<.000, \mathrm{n}=23$. Los informantes con GDS-3 emitieron de media 0,22 metonimias por minuto, los de GDS-4 0,25, los de GDS-5 0,05, los de GDS-6 0 y los de GDS-7 0,02. Si atendemos a los datos distribuidos por estadios de la enfermedad, obtenemos que en el estadio leve la media fue de 0,24 metonimias por minuto, en el estadio moderado esta cifra descendió un 79,2\% (0,05 meto./min.) y en el estadio severo un 16,67\% (0,01 meto./min.). El Gráfico 9 ilustra las metonimias por minuto emitidas por los informantes divididos por grado en la escala GDS. El Gráfico 10 muestra las metonimias por minuto, emitidas por los informantes divididos por estadio de la enfermedad. 


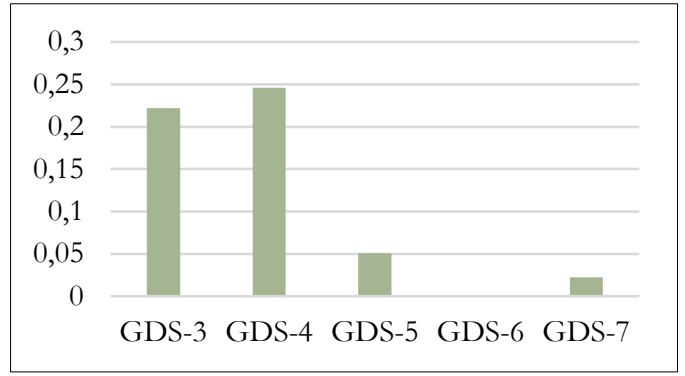

Gráfico 9. Metonimias por minuto emitidas por los informantes clasificados por GDS.

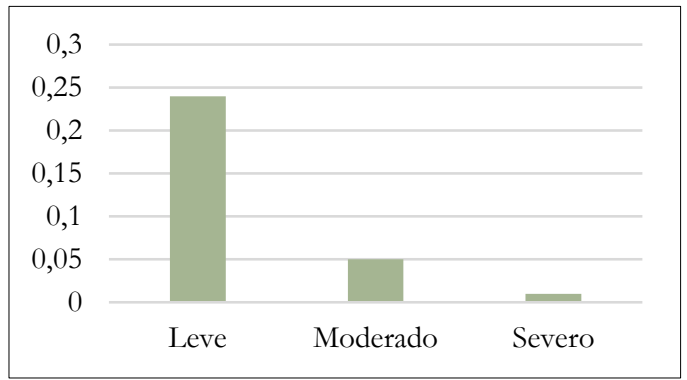

Gráfico 10. Metonimias por minuto emitidas por los informantes clasificados por estadio.

\subsubsection{Metáforas}

Los resultados muestran que existe una correlación negativa débil entre el estadio de la enfermedad (GDS-3 y GDS-4= leve, GDS-5= moderado; GDS-6 y GDS$7=$ severo $)(\mathrm{M}=1,70 ; \mathrm{DE}=, 82)$ y el ratio de metáforas correctas por minuto $(\mathrm{M}=, 30$; $\mathrm{DE}=, 30), r=-, 484, p \leq .019, \mathrm{n}=23$. Los informantes con GDS-3 emitieron de media 0,45 metáforas por minuto, los de GDS-4 0,43, los de GDS-5 0,23, los de GDS-6 0,13 y los de GDS-7 0,04. Si atendemos a los datos distribuidos por estadios de la enfermedad, obtenemos que en el estadio leve la media fue de 0,43 metáforas por minuto, en el estadio moderado esta cifra descendió un 79,2\% (0,23 meta./min.) y en el estadio severo un 16,67\% más (0,08 meta./min.). El Gráfico 11 muestra las metáforas por minuto emitidas por los informantes divididos por grado en la escala GDS. El Gráfico 12 muestra las metáforas por minuto emitidas por los informantes divididos por estadio de la enfermedad. 


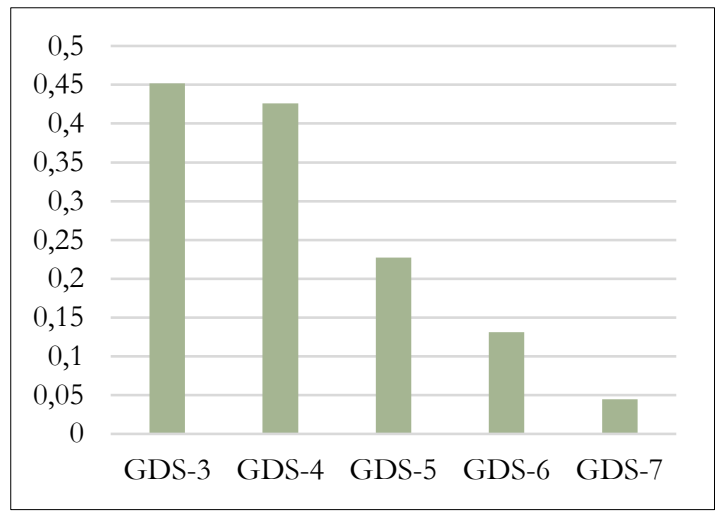

Gráfico 11. Metáforas por minuto emitidas por los informantes clasificados por GDS.

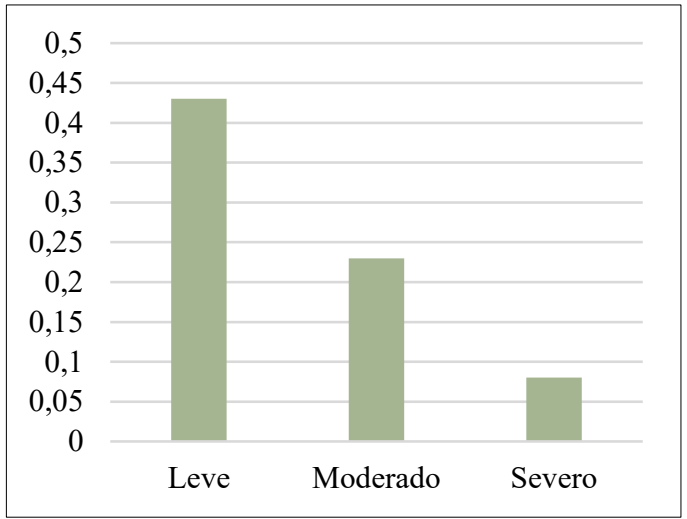

Gráfico 12. Metáforas por minuto emitidas por los informantes clasificados por estadio.

\subsubsection{Lenguaje retórico}

Los resultados muestran que no existe correlación entre el estadio de la enfermedad (GDS-3 y GDS-4= leve, GDS-5= moderado; GDS-6 y GDS-7=severo) $(\mathrm{M}=1.70 ; \mathrm{DE}=.82)$ y la ratio de lenguaje figurado empleado por minuto $(\mathrm{M}=.11$; $\mathrm{DE}=.23), r=-.015, p \leq .946, \mathrm{n}=23$. Los informantes con GDS-3 emitieron de media 0,08 enunciados retóricos por minuto, los de GDS-4 0,07, los de GDS-5 0,29 y tanto los de GDS-6 como los de GDS-7 emitieron 0. Si atendemos a los datos distribuidos por estadios de la enfermedad, obtenemos que en el estadio leve la media fue de 0,07 enunciados retóricos por minuto, en el estadio moderado esta cifra aumentó a 0,29 meta./min. y en el estadio severo un se redujo a 0 meta./min.. El Gráfico 13 recoge los enunciados retóricos por minuto emitidos por los informantes divididos por grado en la escala GDS y el Gráfico 14 muestra los enunciados retóricos por minuto emitidos por los informantes divididos por estadio de la enfermedad. 


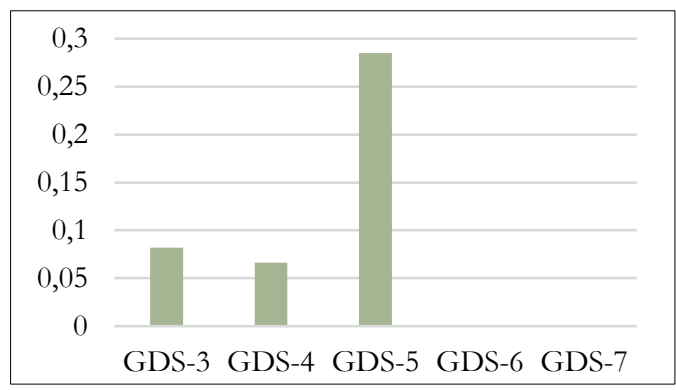

Gráfico 13. Lenguaje retórico por minuto emitido por los informantes clasificados por GDS.

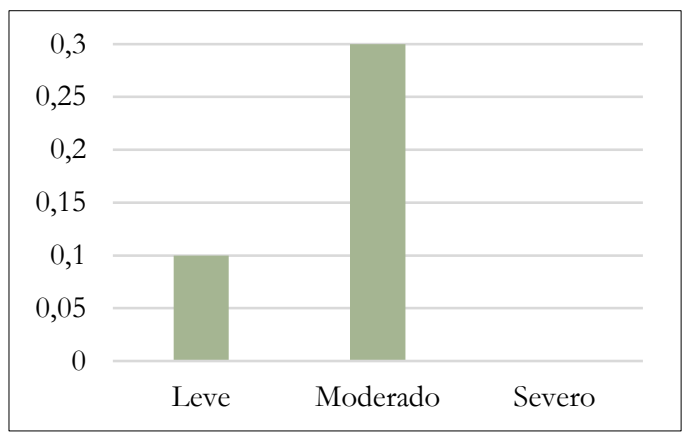

Gráfico 14. Lenguaje retórico por minuto emitido por los informantes clasificados por estadio.

\subsection{El lenguaje figurado como estrategia de compensación}

Los resultados del etiquetado muestran que, efectivamente, el lenguaje figurado puede funcionar como un recurso de compensación, cuando las personas informantes no saben cómo responder en su turno de habla, pero sí que son conscientes de cuál es la estructura de la conversación y que deben emitir un enunciado para mantenerla. En este trabajo, se ha detectado que hay dos formas de lenguaje figurado que cumplen más habitualmente esta función. El primer caso que encontramos es el de los extensores, que frecuentemente se emplean como relleno. En el caso del Ejemplo 1, que mostramos a continuación, la informante I23 está relatando cómo le detectaron una enfermedad a su hija y empieza a enumerar los síntomas. Sin embargo, llega un punto en el que no sabe continuar y recurre al extensor "con esas cosas".

Ejemplo 1

\begin{tabular}{|l|l|}
\hline I23 & -Era ingeniero de caminos \\
\hline INV. & $-¡$ - Caray! \\
\hline I23 & -Sí. Y muy bien. Y entonces empezó así con-con las piernas que le fallaron y con esas cosas y... \\
\hline
\end{tabular}

En el Ejemplo 2, encontramos de nuevo el uso de un extensor como estrategia de compensación. En este caso, la idea de 'si eres buena persona, recibirás cosas buenas' 
que se presenta en la primera línea es repetida por la informante varias veces a lo largo de la conversación. Presumiblemente, se trata de un tema al que recurre para llevar la conversación a un terreno en el que se siente cómoda, por lo que usa el extensor "éche asî" (es asî) como estrategia para reforzar su mensaje.

Ejemplo 2

\begin{tabular}{|l|l|}
\hline I04 & -Se 'tas ben: e eres boa:, pois tamén che han de facer a ti. Tamén. \\
\hline INV. & -Iso é verdade. \\
\hline I04 & -E logo non? Éche así. \\
\hline
\end{tabular}

El segundo tipo de lenguaje figurado que encontramos empleado como estrategia de compensación es el lenguaje retórico. En el caso del Ejemplo 3, la informante no recuerda en qué provincia está su pueblo de nacimiento, por lo que responde irónicamente "mucho para allá en el infierno".

Ejemplo 3

\begin{tabular}{|l|l|}
\hline ENT. & $-¿$ Y dónde nació? \\
\hline I15 & - En... Un pueblo que se llama Barroso. \\
\hline ENT. & $-¿$ Y dónde está? \\
\hline I15 & -Mucho para allá en el infierno. \\
\hline
\end{tabular}

Otra muestra de este recurso la encontramos en el Ejemplo 4. En el caso de este informante, le preguntamos varias veces cuántos hijos tenía y no supo contestar. En una de las ocasiones, para completar el par adyacente empleó dos estrategias de compensación: en primer lugar, una pregunta de confirmación ("fillos?"), a la que recurren en numerosas ocasiones las personas con demencia para ganar tiempo cuando tienen dudas sobre cómo responder (Hamilton, 1994); y, en segundo lugar, encontramos de nuevo el uso de la ironía "eu non, a muller tuvo" (yo no, tuvo mi mujer).

Ejemplo 4

\begin{tabular}{|l|l|}
\hline INV. & -E ten fillos? \\
\hline I15 & -Fillos? \\
\hline INV. & -Hum. \\
\hline I15 & -Eu non, a muller tuvo. (Ríe) \\
\hline
\end{tabular}

\section{Discusión}

Este trabajo ha analizado el uso del lenguaje figurado por parte de personas que han sido diagnosticadas con distintas formas de demencia. Para comenzar, nuestro primer objetivo era identificar los diferentes tipos de lenguaje figurado que las personas con demencia emplean en su discurso. En el caso de nuestro corpus, nos 
hemos encontrado con cinco formas de lenguaje figurado: expresiones fijas, extensores, metáforas, metonimias y lenguaje retórico. Los resultados con respecto a la frecuencia de uso confirman que entre las formas empleadas con mayor frecuencia están las expresiones fijas y los extensores, es decir, formas familiares que normalmente se emplean de manera mecánica.

El trabajo de Papagno (2001) analizó la capacidad de comprensión del lenguaje figurado de las personas con demencia y detectó que aquel que resulta menos problemático es el que emplean y escuchan con más frecuencia. En el caso de la producción, se contempla un fenómeno similar, pues el 47\% de los extensores que anotamos y el $20 \%$ de las metonimias fueron repetidos al menos una vez a lo largo del corpus, mientras que otras formas, como las metáforas $(16 \%)$ y el lenguaje retórico $(9,5 \%)$, tuvieron un porcentaje de repetición más reducido.

En lo que concierne al segundo objetivo del trabajo, verificar si el uso del lenguaje figurado se reduce conforme avanza el deterioro cognitivo, nuestros resultados han determinado que existe una correlación negativa moderada entre el estadio de la enfermedad y el uso del lenguaje figurado $(r=-.600)$. No obstante, al contrario de lo que planteamos en la hipótesis de partida, este declive no se debe a que su uso sea incorrecto, ni estructural ni pragmáticamente, ya que tan solo un 3\% de los enunciados etiquetados está catalogado como error, sino que responde principalmente a un descenso en su frecuencia de uso.

Aparte de esto, cabe destacar que, mientras que en el caso de las expresiones fijas, los extensores discursivos y las metáforas, este deterioro es progresivo durante los tres estadios, el patrón de las metonimias y el lenguaje retórico es diferente. En el caso de las metonimias, se produce un descenso brusco del uso correcto ya entre el estadio leve y moderado (un 79,2\% menos) de la demencia. El caso del lenguaje retórico es más llamativo, pues el estadio moderado es en el que más se usa $(0,29$ veces por minuto en comparación con 0,07 en el estadio leve) y en el estadio avanzado no se utiliza en absoluto.

Esto último podemos atribuirlo en parte a los datos obtenidos al realizar el análisis para el tercer objetivo de este trabajo: detectar cómo el uso del lenguaje figurado puede funcionar en calidad de estrategia compensatoria para mitigar los déficits discursivos causados por la demencia, pues, tal y como apuntan Maclagan et al. (2008), su empleo puede funcionar como estrategia de compensación. Como ya hemos mencionado más arriba, algunos trabajos (Varela Suárez, 2017, 2018) apuntan a que en el estadio moderado es en el que se emplean más estrategias de compensación, pues en el inicial los informantes todavía disponen de suficientes recursos como para continuar con la interacción sin necesidad de recurrir demasiado a la compensación y en el estadio avanzado los hablantes ya no disponen de las capacidades cognitivas suficientes como para emplear estas estrategias de forma eficaz. 
Asimismo, también hemos detectado que los extensores discursivos han sido empleados como estrategia de compensación, sobre todo en el estadio inicial de la enfermedad. Las personas informantes recurren a ellos con frecuencia cuando no saben cómo continuar con una frase o cuando no recuerdan un dato y deciden finalizar el enunciado con una expresión más general como "éche así" o "así es la vida". Sin embargo, aunque no podemos afirmar que esta sea una estrategia exclusiva de los hablantes con demencia, ya que, en general, las personas no diagnosticadas con trastornos cognitivos también recurren a ellas en su praxis comunicativa, la investigación previa apunta a que el uso de tales formas es más recurrente en las personas con deterioro cognitivo (Bridges \& Van Lancker Sidtis, 2013; Zimmerer, et al. 2016). Así, esta parte del trabajo se deberá completar en una futura investigación en la que se analice el uso de estos extensores exclusivamente como estrategia de compensación y se contraste con el uso que hacen de ellos las personas sanas.

Por último, cabe señalar que de este trabajo se deducen futuras líneas de investigación que pueden aportar datos muy valiosos en el campo de la pragmática clínica. Para todas ellas sería necesario recoger una muestra más amplia. En primer lugar sería conveniente recoger más informantes de cada tipo de demencia, pues esto nos permitiría comprobar si el uso del lenguaje figurado tiene características distintas en las diferentes formas de la enfermedad. Además, también nos facilitaría evitar el posible sesgo que se haya podido crear en los resultados debido a la heterogeneidad sintomatológica en la muestra. En segundo lugar, es necesario obtener más informantes de los estadios moderado y avanzado, para así poder disponer de más datos sobre el uso de las figuras retóricas en diferentes condiciones de afectación cognitiva y lingüística. Además, sería recomendable ampliar la representación de las franjas etarias, pues aunque en este trabajo no hemos hallado una correlación significativa entre las variables 'edad' y 'producción de lenguaje figurado', es posible que el uso de las expresiones varíe entre los hablantes de edades distintas. Para finalizar, nuestros datos han apuntado a que nuestros informantes con gallego como primera lengua recurrían ligeramente más al lenguaje retórico como estrategia de compensación. De este modo, si en futuros trabajos aplicamos una perspectiva sociolingüística y ampliamos la muestra de hablantes de ambas lenguas, podremos determinar si se trata de una relación de casualidad o causalidad.

\section{CONCLUSIONES}

De acuerdo con los resultados de este trabajo, la capacidad para emitir enunciados que contengan formas de lenguaje figurado se deteriora conforme avanza el deterioro cognitivo característico de la demencia. Sin embargo, esto se debe a la reducción en la frecuencia de uso y no a un aumento en el uso erróneo de las estructuras. Asimismo, conforme avanza la enfermedad, el uso de los extensores y el lenguaje retórico puede funcionar como una estrategia de compensación que permita mantener la estructura del cambio de turnos cuando la persona con demencia no sabe cómo proseguir con la 
conversación. La investigación futura debe centrarse en recoger una muestra más amplia y variada.

\section{REFERENCIAS BIBLIOGRÁFICAS}

Amanzio, M., Geminiani, G., Leotta, D. \& Cappa, S. (2008). Metaphor comprehension in Alzheimer's disease: Novelty matters. Brain and Language, 107, 1-10.

Bridges, K. A. \& Van Lancker Sidtis, D. (2013). Formulaic Language in Alzheimer's Disease. Aphasiology, 27(7), 799-810.

Brundage, S. B. \& Brookshire, R. H. (1995). A system for scoring proverb interpretations provided by non-brain-damaged adults and aphasic adults. Clinical Aphasiology, 23, 165-177.

Causino Lamar, M. A., Obler, L. K., Knoefel, J. E. \& Albert, M. L. (1994). Communication patterns in end-stage Alzheimer's disease: Pragmatic analysis. En R. L. Bloom, L. K. Obler, S. DeSanti \& J. S. Ehrlich (Eds.) Discourse analysis and applications: Studies in adult clinical populations (pp. 127-235). Hillsdale: Lawrence Erlbaum.

Chapman, S. B., Highley, A. P. \& Thompson, J. L. (1998). Discourse in fluent aphasia and Alzheimer's disease: Linguistic and pragmatic considerations. Journal of Neurolinguistics: Special Issue, 11, 55-78.

Chapman, S. B., Ulatowska, H. K., Franklin, L. R., Shobe, A. E., Thompson, J. L. \& McIntire, D. D. (1997). Proverb interpretation in fluent aphasia and Alzheimer's disease: Implications beyond abstract thinking. Aphasiology, 11(45), 337-350.

Davis, B. H. \& Guendouzi, J. (Eds.) (2013). Pragmatics in dementia discourse. Newcastle upon Tyne: Cambridge Scholars Publishing.

Guendouzi, J. \& Müller, N. (2002). Defining trouble-sources in dementia: Repair strategies and conversational satisfaction in interactions with an Alzheimer's patient. Investigations in clinical phonetics and linguistics, 15-30.

Hamilton, H. E. (1994a). Conversations with an Alqheimer's patient: An interactional sociolinguistic study. Cambridge: Cambridge University Press.

Hamilton, H. E. (1994b). Requests for clarification as evidence of pragmatic comprehension difficulty: The case of Alzheimer's disease. Discourse analysis and applications: Studies in adult clinical populations, 185-199.

Lubinski, R. (Ed.) (1995). Dementia and communication. San Diego: Singular. 
Maclagan, M., Davis, B. \& Lunsford, R. (2008). Fixed expressions, extenders and metonymy in the speech of people with Alzheimer's disease. En S. Granger \& F. Meunier (Eds.), Phraseology: An interdisciplinary perspective (pp. 175-187). Amsterdam/Philadelphia: John Benjamins.

Maki, Y., Yamaguchi, T., Koeda, T. \& Yamaguchi, H. (2013). Communicative competence in Alzheimer's disease: Metaphor and sarcasm comprehension. American Journal of Alzheimer's Disease \& Other Dementias ${ }^{\circledR}, 28(1), 69-74$.

March, E., Wales, R. \& Pattinson, P. (2003). Language use in normal ageing and Dementia of the Alzheimer type. Clinical Psychologist, 7(1), 44-49.

Müller, N. (Ed.) (2006). Multilayered transcription. San Diego, CA: Plural Publishing.

Murgiondo, J. E. \& Tejedor, F. J. T. (2005). Análisis descriptivo de datos en educación (Vol. 2). Madrid: Editorial La Muralla.

Orange, J. B., Lubinski, R. B. \& Higginbotham, D. J. (1996). Conversational repair by individuals with dementia of the Alzheimer's type. Journal of Speech, Language, and Hearing Research, 39(4), 881-895.

Organización Mundial de la Salud (OMS). (2017). Demencia [en línea]. Disponible en: http://www.who.int/mediacentre/factsheets/fs362/es/.

Papagno, C. (2001). Comprehension of metaphors and idioms in patients with Alzheimer's disease: A longitudinal study. Brain, 124(7), 1450-1460.

Pérez Mantero, J. L., (2017). Descripción y análisis pragmático del lenguaje en la demencia de tipo alz̧éimer. Tesis doctoral, Universitat de Valência, Valencia, España.

Perkins, L., Whitworth, A. \& Lesser, R. (1997). Conversation analysis profile for people with cognitive impairment. Londres: Whurr.

Perkins, M. R. (2008). Pragmatic impairment. Cambridge: Cambridge University Press.

Pita Fernández, S. \& Pértega Díaz, S. (1997). Relación entre variables cuantitativas. Cad Aten Primaria, 4, 141-4.

Ripich, D. N., Ziol, E., Fritsch, T. \& Durand, E. J. (2000). Training Alzheimer's disease caregivers for successful communication. Clinical Gerontologist, 21(1), 37-56.

Rousseaux, M., Sève, A., Vallet, M., Pasquier, F. \& Mackowiak-Cordoliani, M. A. (2010). An analysis of communication in conversation in patients with dementia. Neuropsychologia, 48(13), 3884-3890.

Varela Suárez, A. (2017). The question-answer adjacency pair in dementia discourse. International Journal of Applied Linguistics, 1-16. 
Varela Suárez, A. (2018). Lengua y demencia. Diseño y aplicación de un protocolo de demencia discursiva. Tesis doctoral, Universidad de Vigo, Vigo, España.

Vasse, E., Vernooij-Dassen, M., Spijker, A., Rikkert, M. O. \& Koopmans, R. (2010). A systematic review of communication strategies for people with dementia in residential and nursing homes. International Psychogeriatrics, 22(2), 189-200.

Wray, A. (2011). Formulaic language as a barrier to effective communication with people with Alzheimer's disease. Canadian Modern Language Review, 67(4), 429458.

Zimmerer, V. C., Wibrow, M. \& Varley, R. A. (2016). Formulaic language in people with probable Alzheimer's disease: A frequency-based approach. Journal of Alzheimer's Disease, 53(3), 1145-1160

\section{NOTA}

${ }^{1}$ Los casos en los que no disponemos de la información acerca de la edad de las personas participantes han sido marcados con el símbolo (-) en la Tabla. 